\title{
Is radioactive iodine-131 treatment related to the occurrence of non-synchronous second primary malignancy in patients with differentiated thyroid cancer?
}

Marcelo Cruzick de Souza', Denise P. Momesso', Fernanda Vaisman ${ }^{1,2}$, Leonardo Vieira Neto', Rosangela Aparecida Gomes Martins',

Rossana Corbo ${ }^{1,2}$, Mario Vaisman'

\begin{abstract}
Objective: Much controversy relates to the risk of non-synchronous second primary malignancies (NSSPM) after radioactive iodine treatment (RAI-131) in differentiated thyroid cancer (DTC) patients. This study evaluated the relationship between RAI-131 and NSSPM in DTC survivors with long-term follow-up. Materials and methods: Retrospective analysis of 413 DTC cases was performed; 252 received RAI-131 and 161 were treated with thyroidectomy alone. Exclusion criteria were: prior or synchronous non-thyroidal malignancies (within the first year), familial syndromes associated to multiple neoplasms, ionizing radiation exposure or second tumors with unknown histopathology. Results: During a mean follow-up of $11.0 \pm 7.5$ years, $17(4.1 \%)$ patients developed solid NSSPM. Patients with NSSPM were older than those without $(p=0.02)$. RAl-131 and I-131 cumulative activity were similar in patients with and without NSSPM ( $p=0.18$ and $p=0.78$, respectively). Incidence of NSSPM was $5.2 \%$ in patients with RAI-131 treatment and $2.5 \%$ in those without RAI-131 ( $p=0.18$ ). Using multivariate analysis, RAI-131 was not significantly associated with NSSPM occurrence $(p=0.35)$; age was the only independent predictor $(p=0.04)$. Under log rank statistical analysis, after 10 years of follow-up, it was observed a tendency of lower NSSPM-free survival among patients that received RAI-131 treatment ( 0.96 vs. $0.87 ; p=0.06)$, what was not affected by age at DTC diagnosis. Conclusion: In our cohort of DTC survivors, with a long-term follow-up period, RAI-131 treatment and I-131 cumulative dose were not significantly associated with NSSPM occurrence. A tendency of premature NSSPM occurrence among patients treated with RAl-131 was observed, suggesting an anticipating oncogenic effect by interaction with other risk factors. Arch Endocrinol Metab. 2016;60(1):9-15
\end{abstract}

Keywords

Radioactive iodine treatment; differentiated thyroid cancer; secondary primary malignancy
1 Endocrinology Service, Universidade Federal do Rio de Janeiro (UFRJ), Rio de Janeiro, RJ, Brazil

${ }^{2}$ Endocrinology Service, Instituto Nacional do Cancer (Inca) Rio de Janeiro, RJ, Brazil ${ }^{3}$ Research Divison, Hospital Universitário Clementino Fraga Filho (HUCFF), Rio de Janeiro, RJ, Brazil

Correspondence to: Denise P Momesso

Serviço de Endocrinologia Rua Rodolpho Paulo Rocco, 255, $9^{\circ}$ andar

Cidade Universitária - Ilha do Fundão 21941-913 - Rio de Janeiro, RJ, Brazil dmomesso@terra.com.br

Received on May/9/2015 Accepted on June/3/2015

DOI: $10.1590 / 2359-3997000000078$

\section{INTRODUCTION}

$\mathrm{D}$ ifferentiated thyroid carcinoma (DTC), including papillary, follicular and its variants, accounts for over $90 \%$ of all the malignant neoplasms of the thyroid gland (1). DTC incidence has been rising substantially all over the world. The exact reasons for the rising incidence are unclear, but it might be related to better detection by ultrasonography and thyroid fine needle aspiration; improved recognition of histological subtypes; and possible increased exposure to unknown risk factors (2-4). The Brazilian National Cancer Institute (INCa) estimates 9200 new cases of DTC in Brazil in 2014, with an expected incidence (per 100,000 habitants) of 1.15 and 7.89 , among men and women, respectively (5).

Treatment of DTC involves surgery with elective radioactive iodine-131 treatment (RAI-131) in an individualized approach (6-9). Overall prognosis of DTC after treatment is favorable with a low mortality rate (1). Therefore, the great majority of patients with DTC will have a long-term survival and follow-up period. Concerns related to the long-term negative effects of RAI131 are increasing and a more selective use of RAI-131 is being advocated (10). Researchers have noticed non rare cases of non-synchronous second primary malignancies (NSSPM) along the follow-up of non-familial 
DTC, exceptions made to the known syndromes in which DTC is associated with other malignant neoplasms (11). Some authors advocated the relationship between RAI-131 and oncogenic effects (12-16), as suggested by recent studies with DTC patients treated with RAI-131 $(15,16)$. Nevertheless, an Iranian cohort demonstrated that the increased risk of second malignant neoplasms occurred only when the cumulative dose of I-131 exceeded 4.0 GBq (1.08Ci) (17). Furthermore, other studies reported no influence of RAI131 for DTC on the incidence of NSSPM (18).

Therefore, there is still much controversy relating the risk of NSSPM with RAI-131. The aims of this study were to evaluate the relationship between the RAI131 treatment for DTC and the incidence of NSSPM in a cohort of DTC survivors with long-term follow-up, and to evaluate other possible factors associated with NSSPM in DTC survivors.

\section{MATERIALS AND METHODS}

\section{Study population}

From 562 patients with histopathological diagnosis of thyroid cancer treated at the Federal University of Rio de Janeiro between January $1^{\text {st }}$ of 1979 and December 31 of 2009,413 met the inclusion and exclusion criteria. The present study was performed under the research protocol 178/06 approved by local Research Ethics Committee.

Inclusion criteria were DTC confirmed by histopathology and a minimal follow-up of 3 years after surgery. Two hundred and fifty two patients received at least one RAI-131 therapeutic dose, and 161 were submitted to thyroidectomy alone.

Exclusion criteria were: second malignancy prior to or in the first year of the diagnosis of the DTC (classified as synchronous); history of previous exposure to ionizing radiation; familial syndromes known to be related to cancer of other sites; and/or the presence of tumors with unknown histopathological nature which could represent either DTC metastasis or second primary malignancy.

\section{RAl-131 treatment}

Total thyroidectomy was performed in all patients with DTC diagnoses. RAI-131 treatment after surgery respected the international recommendations, and was indicated for patients with tumors larger than 1 centi- meter $(\mathrm{cm})$, multicentricity, capsular or angiolymphatic invasion, extra-thyroidal extension, lymphonodal or distant metastasis and in case of any evidence of postoperative remnant disease on the neck. RAI-131 was administered after levothyroxine withdrawal for 30 days, or, in selected cases, after recombinant TSH administration. Eight days after treatment, patients were submitted to a post-dose whole body scan (WBS), and a therapeutic control WBS was performed 6-12 months after each dose. The standard radioactivity indicated was $100 \mathrm{mCi}$ (3.7GBq); major doses were administered in the presence of lymphonodal or distant metastasis. Subsequent doses were administered in individualized manner, when there was evidence of residual radioactive avid disease.

\section{Follow-up}

All patients were assigned to a regular and periodic follow-up plan for thyroid cancer, which included measurement of serum thyroglobulin and cervical ultrasonography at 6-12 months intervals. Further complementary tests, such as computed tomography and I-131 whole body scan, were performed as an individualized approach whenever clinically indicated. There was no active screening for other malignancies. Only documented non-thyroid neoplasms were considered as NSSPM. NSSPM was diagnosed in the presence of suggestive signs and symptoms followed by complimentary investigation.

\section{Data collection}

For this retrospective analysis, we recorded in a datasheet information of each case including gender, date of diagnosis, age at diagnosis, histophatological subtypes of DTC, the first dose and the subsequent cumulative I-131 dose for the group treated with RAI-131, followup period, data about the NSSPM (age at diagnosis, subtype) and cause of death (when applicable).

\section{Outcomes}

Primary outcomes of the study were to determine the incidence of NSSPM and to evaluate the association of NSSPM with RAI-131. Secondary outcomes were to evaluate other possible factors associated with NSSPM in patients with DTC.

The endpoint of the study was determined for each patient as the date of diagnosis of NSSPM, death, loss of follow-up or the closing date of data collection (December 31, 2012), whichever occurred first. 
A first analysis was performed with the entire population in order to evaluate the incidence of NSSPM. Patients where then distributed into two groups for analysis: with and without NSSPM. We aimed to evaluate clinical factors associated with NSSPM in DTC patients.

In order to evaluate the incidence of NSSPM in patients treated with RAI-131 a separate analysis was performed which distributed patients in two groups: with and without RAI-131 treatment.

At last, patients who developed NSSPM were separately analyzed. Clinical characteristics and the exposure impact of RAI-131 in these patients were evaluated.

\section{Statistical analysis}

Statistical analysis was processed using SPSS for Windows 13.0 (SPSS Inc. 2004). Under univariate analysis to compare between groups, categorical variables were evaluated by Fisher or chi-square tests, and ordinal variables were evaluated by Mann-Whitney. Multivariate analysis was performed by logistic regression analysis (enter method). Endpoint-free cumulative probability was defined, for each group, using Kaplan-Meier method, and comparison between curves was obtained by log-rank (Mantel-Cox) statistics. Significance criteria were defined in $5 \%(\mathrm{p}<0.05)$.

\section{RESULTS}

Baseline characteristics of the entire population $(\mathrm{n}=$ 413) described in table 1 were: mean age of $44.1 \pm$ 15.3 years, $362(87.6 \%)$ women, $309(74.8 \%)$ papillary carcinoma subtype, and $252(61 \%)$ patients were submitted to RAI-131. Mean follow-up period since diagnosis was $11.0 \pm 7.5$ years. In the end of the follow-up, $6(1.4 \%)$ patients died, 2 of them due to thyroid cancer (specific mortality rate $0.48 \%$ ). During the follow-up period, 17 (4.1\%) patients developed NSSPM.

Clinical characteristics of patients with and without NSSPM are demonstrated in table 2. It was observed significant difference of age on DTC diagnosis. Gender, histological type, RAI-131 treatment and I-131 cumulative activity were similar in both groups.

Multivariate analysis by logistic regression was performed to evaluate simultaneous influence of independent variables on the occurrence of NSSPM (Table 3). Age at diagnosis was the only significant predicting factor independently associated to NSSPM occurrence ( $\mathrm{p}$ $=0.04)$. RAI-131 was not significantly associated with NSSPM occurrence $(\mathrm{p}=0.35)$.
Table 1. Baseline characteristics of the entire population of differentiated thyroid cancer patients studied $(n=413)$

\begin{tabular}{lc}
\hline Baseline variables & $\begin{array}{c}\text { DTC patients } \\
\mathbf{N}=\mathbf{4 1 3}\end{array}$ \\
\hline Age at diagnosis of DTC (years) & $44.1( \pm 15.3)$ \\
Gender & \\
Female & $362(87.6 \%)$ \\
$\quad$ Male & $51(12.4 \%)$ \\
Histological type & \\
Papillary & $309(74.8 \%)$ \\
Follicular & $104(25.2 \%)$ \\
RAl-131 & $252(61.0 \%)$ \\
Follow-up period since diagnosis of DTC (years) & $11.0( \pm 7.5)$ \\
\hline
\end{tabular}

Values are represented by mean $( \pm S D)$, number of patients (\%) or median (range). DTC: differentiated thyroid carcinoma; RAI-131: I ${ }^{131}$ radioactive iodine treatment.

Table 2. Descriptive analysis of groups of DTC patients with and without NSSPM

\begin{tabular}{lccc}
\hline Baseline variables & $\begin{array}{c}\text { With } \\
\text { NSSPM } \\
\text { (n= 17) }\end{array}$ & $\begin{array}{c}\text { Without } \\
\text { NSSPM } \\
\text { (n= 396) }\end{array}$ & P value \\
\hline $\begin{array}{l}\text { Age at diagnosis of DTC (years) } \\
\text { Mean }( \pm \text { SD) }\end{array}$ & $52( \pm 12)$ & $44( \pm 15)$ & $0.02^{*}$ \\
Gender & & & \\
$\quad$ Female & $14(82.4 \%)$ & $348(87.9 \%)$ & 0.35 \\
$\quad$ Male & $3(27.6 \%)$ & $48(12.1 \%)$ & \\
Histological type & & & \\
$\quad$ Papillary & $13(76.5 \%)$ & $306(77.3 \%)$ & 0.56 \\
$\quad$ Follicular & $4(23.5 \%)$ & $90(22.7 \%)$ & \\
RAl-131 & $13(76.5 \%)$ & $239(60.4 \%)$ & 0.18 \\
I-131 cumulative activity (mCi) & $150(800)$ & $150(1400)$ & 0.78 \\
$\begin{array}{l}\text { Follow-up period since } \\
\text { diagnosis of DTC (years) }\end{array}$ & $5.3(37.1)$ & $9.0(26.2)$ & 0.07 \\
\hline
\end{tabular}

Values are represented by mean $( \pm S D)$, number of patients (\%) or median (range). DTC: differentiated thyroid carcinoma; RAl-131: / 131 radioactive iodine treatment; NSSPM: nonsynchronous second primary malignancy.

* Statistically significant $p<0.05$.

Table 3. Multivariate analysis by logistic regression (enter method) to evaluate factors independently associated with NSSPM occurrence in DTC patients

\begin{tabular}{lccc}
\hline Variable & Coefficient & S.E. & P value \\
\hline Age at DTC diagnosis (years) & 0.04 & 0.17 & $0.04^{*}$ \\
Gender (Female) & -0.56 & 0.68 & 0.41 \\
Histological type (Papillary) & 0.03 & 0.60 & 0.97 \\
RAl-131 & 0.56 & 0.59 & 0.35 \\
\hline
\end{tabular}

DTC: differentiated thyroid carcinoma; NSSPM: non-synchronous second primary malignancy; RAl-131: I $^{131}$ radioactive iodine treatment; S.E.: standard error.

${ }^{*}$ Statistically significant $p<0.05$.

The incidence of NSSPM in patients with and without RAI-131 treatment is demonstrated in figure 1. There was no statistically difference between the groups, since the incidence of NSSPM in patients that received RAI- 131 was of $5.2 \%$ and in those that did not receive RAI- 131 was of $2.5 \%(\mathrm{p}=0.18)$. 


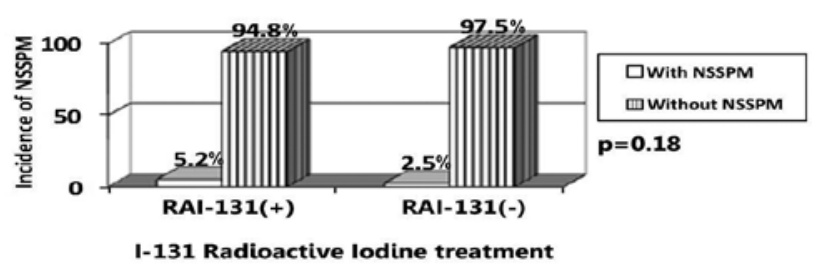

Figure 1. Incidence of NSSPM in DTC patients treated with and without RAI-131. NSSPM: non-synchronous second primary malignancy; DTC: differentiated thyroid cancer; RAl-131: I $^{131}$ radioactive iodine treatment.

The individual characteristics of the 17 patients who developed NSSPM are described in table 4. In order to evaluate the RAI-131 exposure impact on NSSPM occurrence of these patients, the endpoint-free survival cumulative probability was calculated by Kaplan-Meier method for patients with NSSPM treated with and without RAI-131 and, consecutively, endpoint-free survival probability curves were compared by log rank statistical analysis. Patients with NSSPM treated with RAI-131 had an endpoint-free survival probability ratio of 0.96 or $96 \%$ and those treated without RAI-131 of 0.87 or $87 \%$ $(\mathrm{p}=0.06)$ (Figure 2A). Thus, assuming the increased risk exposure time for the RAI-131 treated patients as the time course since the first dose until the endpoint (NSS-
PM occurrence), after 10 years of follow-up it was observed a tendency of lower endpoint-free survival among RAI-131 treated subjects (Figure 2A). Considering the difference of age at DTC diagnosis between groups, we proposed to evaluate Kaplan-Meier endpoint-free curves stratified by age range ( 45 years cut-off) and it was not observed significant difference on age-adjusted log rank statistical analysis $(\mathrm{p}=0.18)$ (Figure $2 \mathrm{~B})$.

\section{DISCUSSION}

DTC incidence is increasing worldwide and given its overall good prognosis, an uprising number of DTC survivors will be followed for a long-time period. Concerns relating to the occurrence of NSSPM in DTC survivors and its relation to RAI-131 are increasing. In our study, we evaluated DTC survivors for a long-time period, aiming the occurrence of NSSPM and its relationship with RAI-131 treatment. In our cohort of DTC survivors, an incidence of $4.1 \%$ of NSSPM was observed and RAI-131 treatment was not significantly associated with NSSPM, although a tendency to a lower NSSPMfree survival was observed, suggesting an anticipating role of RAI-131 exposure on the NSSPM occurrence.

Table 4. Individual characteristics of patients with DTC that developed NSSPM

\begin{tabular}{ccccccccc}
\hline Gender & $\begin{array}{c}\text { DTC } \\
\text { histological } \\
\text { subtype }\end{array}$ & $\begin{array}{c}\text { Age at DTC } \\
\text { diagnosis } \\
\text { (years) }\end{array}$ & $\begin{array}{c}\text { Age at } \\
\text { NSSPM } \\
\text { diagnosis } \\
\text { (years) }\end{array}$ & RAl-131 & $\begin{array}{c}\text { Cumulative } \\
\text { I-131 activity } \\
\text { (mCi) }\end{array}$ & $\begin{array}{c}\text { Time period } \\
\text { from RAl-131 } \\
\text { and NSSPM } \\
\text { (years) }\end{array}$ & $\begin{array}{c}\text { NSSPM } \\
\text { Histological type }\end{array}$ \\
\hline 1 & F & FTC & 36.4 & 61.1 & Yes & 100 & 4.3 & Adenocarcinoma" \\
2 & F & PTC & 42.2 & 69.2 & Yes & 100 & 16.8 & Skin (melanoma) \\
3 & M & FTC & 68.2 & 78.5 & Yes & 620 & 9.7 & Prostate \\
4 & F & FTC & 68.1 & 69.8 & Yes & 150 & 1.1 & Breast \\
5 & F & PTC & 52.5 & 61.5 & Yes & 300 & 8.9 & Uterus \\
6 & F & PTC & 59.3 & 68.3 & Yes & 100 & 8.1 & Skin (BCC) \\
7 & F & PTC & 64.8 & 75.0 & Yes & 350 & 9.8 & Breast \\
8 & F & PTC & 48.6 & 53.9 & Yes & 350 & 4.0 & Gastric \\
9 & F & PTC & 48.0 & 52.4 & Yes & 100 & 3.7 & Uterus \\
10 & F & PTC & 76.5 & 79.3 & Yes & 150 & 2.6 & Breast \\
11 & F & PTC & 59.1 & 72.3 & Yes & 900 & 12.8 & Breast \\
12 & M & FTC & 53.3 & 55.3 & Yes & 150 & 2.0 & Skin (SCC) \\
13 & F & PTC & 42.1 & 46.5 & Yes & 100 & 3.8 & Lungs \\
14 & F & PTC & 39.4 & 41.9 & No & - & - & Breast \\
15 & F & FTC & 43.3 & 56.7 & No & - & - & Skin (BCC) \\
16 & M & PTC & 49.1 & 54.1 & No & - & - & Prostate \\
17 & F & PTC & 39.3 & 44.0 & No & - & - & Breast \\
\hline
\end{tabular}

DTC: differentiated thyroid carcinoma; NSSPM: non-synchronous second primary malignancy; RAl-131= $\left.\right|^{131}$ radioactive iodine treatment; F: female; M: male; PTC: papillary thyroid carcinoma; FTC: follicular thyroid carcinoma; BCC: basal cell cancer; SCC: squamous cell cancer. \#Adenocarcinoma indicates glandular epithelium neoplasm from unknown site other than thyroid cancer, excluded by immunohistochemical study. 
A

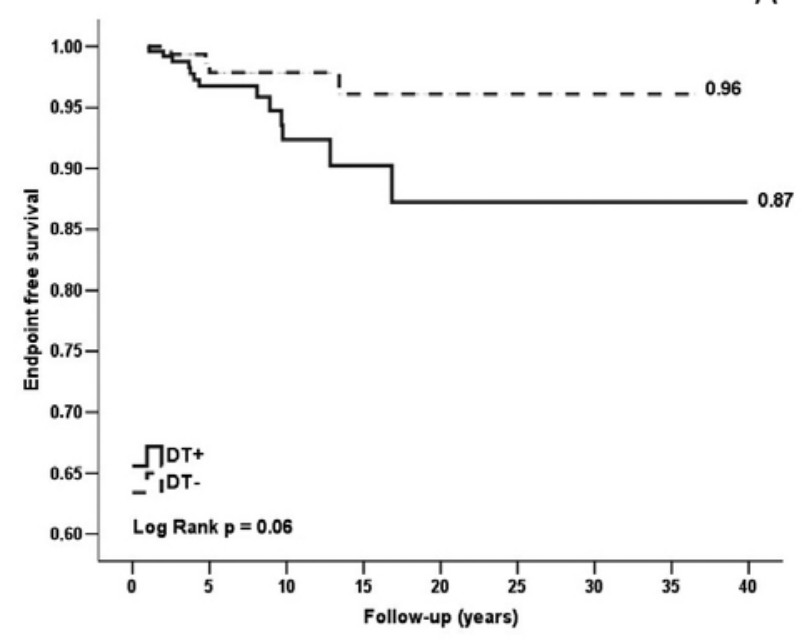

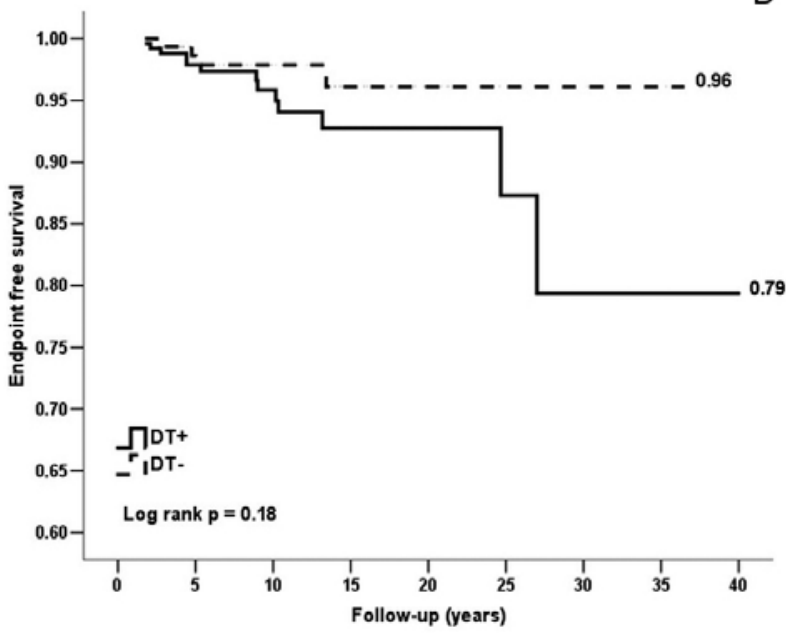

Figure 2. Endpoint-free cumulative probability curves of NSSPM. (A) Global endpoint-free survival. (B) Age-adjusted endpoint-free survival (45 years cutoff). NSSPM: non-synchronous second primary malignancy; DTC: differentiated thyroid cancer; RAl-131(+): DTC patients treated with I-131 radioactive iodine; RAl-131(-): DTC patients not treated with I-131 radioactive iodine.

A greater risk of second primary malignancies among DTC survivors in comparison to general population has been shown by previous studies $(19,20)$. The incidence of $4.1 \%$ cases of NSSPM in our cohort is in accordance to the previous literature description of $1.2 \%-8.9 \%(17,18,21,22)$. It has been described an increased risk among DTC survivors for secondary malignancies of salivary glands, stomach, breast, central nervous system, colorectal, prostate, genitourinary tract, kidney, adrenal gland, bone and joints, soft tissue sarcoma and hematological cancers (23). In this study, we found only solid NSSPM and the most frequent site was breast; followed by skin and prostate; uterus, gastrointestinal and lung cancer were also described. The association between breast cancer and DTC has been described by previous studies both as non-synchronous and synchronous tumors, as well as the association with auto-immune thyroid disorders $(21,22,24)$.

Many factors may account for the elevated risk of secondary malignancies in DTC survivors. Environmental and genetic mechanisms may play an important role, since many tumors exhibit similar genetic profiles to DTC. Furthermore, much controversy exists about whether RAI-131 affects this endpoint. Data on RAI131 influence on secondary malignancies are confounding and it depends on the type of secondary tumor analyzed $(15,21,22)$.

In the present work, during a long-term follow-up of $11.0 \pm 7.5$ years, no significant association was found between RAI-131 and the overall occurrence of solid NSSPM in DTC survivors. Similarly, previous studies, despite the lower follow-up period, also have described that the overall rate of NSSPM was not increased by RAI-131, although the hazard of event may be increased in patients that received higher activities $(17,18,20)$. This dose-dependent effect was not observed in our study, what could be due to the relative small number of patients who developed NSSPM. Furthermore, the overall cumulative I-131 activity of the patients included in this study was low and evidence in the literature indicates that the chance of NSSPM is increased in patients who had received a cumulative activity of I-131 exceeding $40 \mathrm{GBq}$ (1.08 Ci) (17).

A meta-analysis by Sawka and cols. demonstrated a slight increase in the polled estimate risk for NSSPM in DTC survivors that received RAI-131, with a $1 \%$ greater absolute risk in the RAI-131 treated than in non-treated DTC survivors. Notably, it demonstrated a specific increased risk of leukemia incidence, but no significant increased risks of other solid malignancies (15). Similar findings were reported by Brown and cols., with data from the largest cohort of DTC survivors, describing an increased overall risk of NSSPM among DTC survivors, and an increased specific risk of leukemia and stomach cancer in RAI-131 treated patients (22). Instead, Rubino and cols. described a linear dose-response relationship among RAI-131 and the occurrence of leukemia and only some specific solid malignancies (colorectal, salivary glands, bone, soft tissue cancer), with no association with others solid malignancies (breast, kidney, central nervous system) (21). In our cohort of DTC survivors, we detected only solid tumors and no 
cases of leukemia were diagnosed during the follow-up, what might explain the lack of association between RAI-131 and NSSPM. Furthermore, the main NSSPM found was breast cancer, which has been associated with DTC and previous studies have corroborated no influence of RAI-131 on its development $(21,22)$.

An interesting finding of our study was a statistical tendency of RAI-131 impact on anticipating NSSPM occurrence in DTC survivors, considering the endpoint-free cumulative probability calculated by Kaplan-Meier method, and this effect was more noticeable after 10 years. This may suggest that RAI-131, despite not directly associated with the development of NSSPM, may interact with other genetic and environmental factors leading to premature occurrence of NSSPM. Still, given that our mean follow-up period was of 11.0 \pm 7.5 years and the small number of patients with NSSPM that did not receive RAI-131 treatment, further studies with longer follow-up period and a larger cohort of patients will be needed to confirm this finding. Furthermore, we cannot rule out a surveillance bias, in which patients with DTC and RAI-131 were submitted to closer follow-up and diagnosed second tumors earlier.

This study also evaluated variables other than RAI131 that could be associated with NSSPM, such as age, gender and DTC histological subtype. Age at DTC diagnosis was the only independent factor with significantly associated with NSSPM, corroborating findings of previous studies $(16,25)$. Older age was observed in patients with DTC that developed NSSPM.

It is also important to address that, given our study criteria, 15 patients were excluded because of other primary malignant neoplasms prior or synchronous to DTC. This can be comparable to the results of one Dutch and one Turkish studies in which approximately half of the patients had thyroid cancer not in the first place, but after another malignant neoplasm, what suggests common mechanisms other than causal relation $(20,26)$. In the same way, it was noticed similar frequency of second malignancy both before and after RAI-131 in a subset of Brazilian DTC patients studied by Hirosawa and cols. (18).

Our study strengths rely on a long-term follow-up of a cohort with significant numbers of DTC survivors that included patients with and without RAI-131, carefully surveyed. Nevertheless, the study has limitations inherent to its retrospective design, which includes the inability to infer a cause-effect relationship and the pro- bability of selection bias. Furthermore, no active investigation for secondary malignances was performed and only clinically apparent disease was diagnosed; thus the possibility of silent NSSPM not diagnosed must be considered.

\section{CONCLUSIONS}

In our cohort of DTC survivors, followed by a longterm period, an incidence of $4.1 \%$ NSSPM was observed, related to the occurrence of solid malignancies. We did not found a statically significant association between RAI-131 treatment or cumulative I-131 activity and NSSPM occurrence. Patients with more advanced age at DTC diagnoses were under greater risk of NSSPM development. This study provides further support that the role of RAI-131 on NSSPM occurrence might be related to the type of secondary malignancy, with no evidence of increased risk of solid malignancies related to RAI-131 in our cohort of DTC survivors followed by a long time period.

Interestingly, our study suggests a statistical tendency of premature NSSPM occurrence among patients treated with RAI-131, despite the small number of DTC patients with NSSPM. This finding indicates that RAI-131 may play a role on anticipating NSSPM occurrence by interacting with many other risk factors involved in the oncogenesis, such as genetics and environmental factors. Further studies to evaluate this issue will be necessary.

Fundings: this research did not receive any specific grant from any funding agency in the public, commercial or not-for-profit section.

Disclosure: no potential conflict of interest relevant to this article was reported.

\section{REFERENCES}

1. HundahI SA, Fleming ID, Fremgen AM, Menck HR. A National Cancer Data Base report on 53,856 cases of thyroid carcinoma treated in the U.S., 1985-1995 [see comments]. Cancer. 1998;83(12): 2638-48.

2. Enewold L, Zhu K, Ron E, Marrogi AJ, Stojadinovic A, Peoples $\mathrm{GE}$, et al. Rising thyroid cancer incidence in the United States by demographic and tumor characteristics, 1980-2005. Cancer Epidemiol Biomarkers Prev. 2009;18(3):784-91.

3. Davis L, Welch HG. Increasing incidence of thyroid cancer in the United States, 1973-2002. JAMA. 2006;295(18):2164-7.

4. Burgess JR, Tucker P. Incidence trends for papillary thyroid carcinoma and their correlation with thyroid surgery and thyroid fineneedle aspirate cytology. Thyroid. 2006;16(1):47-53. 
5. Instituto Nacional do Câncer José Alencar Gomes da Silva, Estimativa 2014. Incidência de Câncer no Brasil. Estimativas. Available at: <http://www.inca.gov.br/estimativa/2014/tabelaestados. asp?UF=BR>. Accessed on: Jan 18, 2014.

6. American Thyroid Association (ATA) Guidelines Taskforce on Thyroid Nodules and Differentiated Thyroid Cancer; Cooper DS, Doherty GM, Haugen BR, Kloos RT, Lee SL, Mandel SJ, et al. Revised American Thyroid Association management guidelines for patients with thyroid nodules and differentiated thyroid cancer. Thyroid. 2009;19(11):1167-214.

7. Maia AL, Ward LS, Carvalho GA, Graf H, Macie RMB, Maciel LMZ, et al. Nódulos de tireoide e câncer diferenciado de tireoide: consenso brasileiro. Arq Bras Endocrinol Metab. 2007;51(5):867-3.

8. Pacini $F$, Schlumberger $M$, Dralle $H$, Elisei $R$, Smit JW, Wiersinga W; European Thyroid Cancer Taskforce. European consensus for the management of patients with differentiated thyroid carcinoma of the follicular epithelium. Eur J Endocrinol. 2006;154(6):787803.

9. Pitoia F, Ward L, Wohllk N, Friguglietti C, Tomimori E, Gauna A, et al. Recommendations of the Latin American Thyroid Society on diagnosis and management of differentiated thyroid cancer. Arq Bras Endocrinol Metabol. 2009;53(7):884-7.

10. Tuttle RM. Risk-adapted management of thyroid cancer. Endocr Pract. 2008;14(6):764-74.

11. Nosé V. Familial follicular cell tumors: classification and morphological characteristics. Endocr Pathol. 2010;21(4):219-26.

12. Hall PF. Cancer risks after medical radiation. Med Oncol Tumor Pharmacother. 1991;8(3):141-5.

13. Dottorini ME, Lomuscio G, Mazzucchelli L, Vignati A, Colombo L. Assessment of female fertility and carcinogenesis after iodine-131 therapy for differentiated thyroid carcinoma. J Nucl Med. 1995;36(1):21-7.

14. de Vathaire F, Schlumberger M, Delisle MJ, Francese C, Challeton $C$, de la Genardiére $E$, et al. Leukaemias and cancers following iodine-131 administration for thyroid cancer. $\mathrm{Br} \mathrm{J}$ Cancer. 1997;75(5):734-9.

15. Sawka AM, Thabane L, Parlea L, Ibrahim-Zada I, Tsang RW, Brierley JD, et al. Second primary malignancy risk after radioactive iodine treatment for thyroid cancer: a systematic review and meta-analysis. Thyroid. 2009;19(5):451-7.
16. Lang BH, Wong IO, Wong KP, Cowling BJ, Wan KY. Risk of second primary malignancy in differentiated thyroid carcinoma treated with radioactive iodine therapy. Surgery. 2012;151(6):844-50.

17. Fallahi $B$, Adabi $K$, Majidi M, Fard-Esfahani $A$, Heshmat $R$, Larijani $B$, et al. Incidence of second primary malignancies during a long-term surveillance of patients with differentiated thyroid carcinoma in relation to radioiodine treatment. Clin Nucl Med. 2011;36(4):277-82.

18. Hirosawa RM, Marivo M, Luengo JML, Tagliarini JV, Castilho EC, Marques MA, et al. Does radioiodine therapy in patients with differentiated thyroid cancer increase the frequency of another malignant neoplasm? ISRN Oncol. 2011;2011:708343.

19. Sandeep TC, Strachan MW, Reynolds RM, Brewster DH, Scélo G, Pukkala $E$, et al. Second primary cancers in thyroid cancer patients: a multinational record linkage study. J Clin Endocrinol Metab. 2006;91(5):1819-25.

20. Verkooijen RB, Smit JW, Romijn JA, Stokkel MP. The incidence of second primary tumors in thyroid cancer patients is increased, but not related to treatment of thyroid cancer. Eur J Endocrinol. 2006;155(6):801-6.

21. Rubino C, de Vathaire F, Dottorini ME, Hall P, Schvartz C, Couette $\mathrm{JE}$, et al. Second primary malignancies in thyroid cancer patients. Br J Cancer. 2003;89(9):1638-44.

22. Brown AP, Chen J, Hitchcock YJ, Szabo A, Shrieve DC, Tward JD. The risk of second primary malignancies up to three decades after the treatment of differentiated thyroid cancer. J Clin Endocrinol Metab. 2008;93(2):504-15.

23. Subramanian S, Goldstein DP, Parlea L, Thabane L, Ezzat S, Ibrahim-Zada I, et al. Second primary malignancy risk in thyroid cancer survivors: a systematic review and meta-analysis. Thyroid. 2007;17(12):1277-88.

24. Giani C, Fierabracci P, Bonacci R, Gigliotti A, Campani D, De Negri $F$, et al. Relationship between breast cancer and thyroid disease: relevance of autoimmune thyroid disorders in breast malignancy. J Clin Endocrinol Metab. 1996;81(3):990-4.

25. Berthe E, Henry-Amar M, Michels JJ, Rame JP, Berthet P, Babin $E$, et al. Risk of second primary cancer following differentiated thyroid cancer. Eur J Nucl Med Mol Imaging. 2004;31(5):685-91.

26. Omür O, Ozcan Z, Yazici B, Akgün A, Oral A, Ozkiliç H. Multiple primary tumors in differentiated thyroid carcinoma and relationship to thyroid cancer outcome. Endocr J. 2008;55(2):365-72. 